\title{
ANÁLISE ESTRUTURAL EM FLORESTA OMBRÓFILA DENSA DE TERRA FIRME NÃO EXPLORADA, AMAZÔNIA ORIENTAL ${ }^{1}$
}

\author{
Deoclides Ricardo de Souza², Agostinho Lopes de Souza ${ }^{3}$, Helio Garcia Leite ${ }^{3}$ e Jorge Alberto Gazel Yared ${ }^{4}$
}

\begin{abstract}
RESUMO - O estudo teve como objetivo analisar a composição florística e as estruturas horizontal, interna e diamétrica da floresta. A pesquisa foi conduzida na Unidade de Manejo Florestal (UMF) da Fazenda Tracajás (02³5’53”'S e 4747’10”W), Município de Paragominas, Estado do Pará, Brasil. A floresta foi estratificada em três áreas homogêneas, denominadas classes I, II e III de estoques volumétricos, empregando-se análise multivariada: análises de agrupamento e discriminante. Em cada classe de estoque foram instaladas, aleatoriamente, cinco parcelas de 100 x $100 \mathrm{~m}$ (1,0 ha) cada uma, para medição dos indivíduos com dap $\geq 15 \mathrm{~cm}$. No centro de cada parcela de 100 × 100 m foi instalada uma subparcela de 10 × 100 m (0,1 ha), para medição dos indivíduos com $5 \mathrm{~cm} \leq$ dap $<15 \mathrm{~cm}$. Nas classes I, II e III de estoques volumétricos das árvores com dap $\geq 15 \mathrm{~cm}$ (nível I de inclusão), respectivamente, estimou-se uma densidade total de 322,4; 309,0; e 313,8 indivíduos por hectare, bem como dominância total de 27,36; 27,45; e 25,88 m²/ha e volume de fuste total de 358,69; 328,33; e $308,69 \mathrm{~m}^{3} / \mathrm{ha}$. Nas classes I, II e III de estoques volumétricos das árvores com $5 \mathrm{~cm} \leq$ dap $<15 \mathrm{~cm}$ (nível II de inclusão), respectivamente, estimaram-se densidade total de 846; 854; e 886 indivíduos por hectare, dominância total de 4,80; 4,93; e 5,46 m²/ha e volume de fuste total de 93,98; 91,23; e 97,61 $\mathrm{m}^{3} / \mathrm{ha}$. As espécies de maior valor de importância relativa, Lecythis idatimon (potencial), Rinorea guianensis (não comercial) e Pouteria guianensis (potencial), ocorreram em todos os níveis de inclusão e classes de estoque. As distribuições de diâmetros de todas as espécies e das espécies comerciais com dap $\geq 5 \mathrm{~cm}$, estimadas pela equação de Meyer, confirmaram a tendência exponencial negativa (“J-invertido”). A análise da estrutura da floresta em classes de estoque permitiu melhor conhecimento da composição de espécies e da estrutura fitossociológica, sendo útil na tomada de decisões em planos de manejo de rendimento sustentável.
\end{abstract}

Palavras-chave: Floresta tropical, diversidade, composição florística e estrutura fitossociológica.

\section{STRUCTURAL ANALYSIS IN AN UNEXPLORED TERRA FIRME DENSE OMBROPHYLOUS FOREST, EASTERN AMAZON}

\begin{abstract}
The objective of this study was to analyze the floristic composition and the horizontal, internal and diametric structure of an unexplored terra firme dense ombrophylous forest. The research was carried out at the Forest Management Unit (FMU) in Tracajás Farm (02'35'53" S and 47 47'10"W), owned by Nova Era Agroflorestal, in Paragominas, Pará, Brazil. The forest was stratified in three homogeneous areas denominated classes of volumetric stock I, II and III, through multivariate analysis (cluster and discriminant analysis). In each of the stock classes, five plots of $100 \times 100 \mathrm{~m}(1 \mathrm{ha})$ were randomly set up for the measurement of individuals with $\mathrm{dbh} \geq 15 \mathrm{~cm}$. In the center of each $100 \times 100 \mathrm{~m}$ plot, a subplot of $10 \times 100 \mathrm{~m}(0.1 \mathrm{ha})$ was installed for the measurement of individuals with $5 \mathrm{~cm} \leq \mathrm{dbh}<15 \mathrm{~cm}$. In classes I, II and III of trees
\end{abstract}

\footnotetext{
${ }^{1}$ Recebido em 21.10.2003 e aceito para publicação em 10.11.2005.

${ }^{2}$ Departamento de Engenharia Agronômica da Universidade Federal de Sergipe - UFS. E-mail:<souzadr@hotmail.com>.

${ }^{3}$ Departamento de Engenharia Florestal da Universidade Federal de Viçosa. E-mail: <alsouza@mail.ufv.br>.

${ }^{3}$ EMBRAPA-CPATU, Belém, Pará.
} 
with $\mathrm{dbh} \geq 15 \mathrm{~cm}$ (level I inclusion), the total density was estimated at 322.4; 309.0 and 313.8 individuals per hectare, respectively. The total dominance was of 27.36; 27.45 and $25.88 \mathrm{~m}^{2} /$ ha and the total volume of commercial whole-tree were of 358.69; 328.33 and $408.69 \mathrm{~m}^{3} / \mathrm{ha}$. In classes I, II and III of individuals with $5 \mathrm{~cm} \leq \mathrm{dbh}<15 \mathrm{~cm}$ (level II inclusion), the total density was estimated at 846; 854 and 886 individuals per hectare, respectively. The total dominance was of 4.80; 4.93 and of $5.46 \mathrm{~m}^{2} / \mathrm{ha}$ and the total volume of commercial whole-tree were of 93.98; 91.23 and $97.61 \mathrm{~m}^{3} / \mathrm{ha}$. The species of highest relative importance value, Lecythis idatimon (potential), Rinorea guianensis (non-commercial) and Pouteria guianensis (potential) occurred in all inclusion levels and all three stock classes. The diametric distribution of all species and of the commercial species with $\mathrm{dbh} \geq 5 \mathrm{~cm}$ estimated by the Meyer equation confirmed the negative exponential trend ("inverted $J$ "). The analysis of the forest structure in stock classes allowed the evaluation of the species composition and the forests phytosociologic structure, being useful for the elaboration and execution of sustainable management plans.

Keywords: Tropical forest, diversity, floristic composition and phytosociologic structure.

\section{INTRODUÇÃO}

A maioria das florestas tropicais nativas da Amazônia tem sido explorada de forma não sustentável, sem aplicação dos critérios de sustentabilidade do manejo florestal, o que caracteriza perda da cobertura florestal e da diversidade de espécies, antes mesmo que se tenha o conhecimento dessa riqueza natural.

Qualquer intervenção planejada em determinada floresta natural tem de ser precedida de inventário minucioso, que forneça estimativas fidedignas dos parâmetros: diversidade, freqüência, densidade, dominância e as distribuições diamétrica e espacial das espécies, bem como os valores ecológico, econômico e social das espécies. As florestas têm de ser tratadas caso a caso, ou seja, individualmente. Para Souza (2003), a análise da estrutura das florestas nativas deve se iniciar com uma abordagem da composição florística e das estruturas horizontal, vertical, interna e paramétrica, contemplando as estimativas de distribuição de diâmetros, área basal e volume. Segundo Bourgeron (1983), o conceito de estrutura se relaciona com o de diversidade, principalmente quando se procura uma explicação para o grande número de espécies existentes nas florestas tropicais naturais.

A diversidade de uma floresta abrange dois diferentes conceitos: riqueza e eqüidade. Riqueza referese ao número de espécies em uma comunidade; eqüidade, à forma pela qual os números de indivíduos são distribuídos entre as espécies (CARVALHO et al., 1999). A freqüência aponta a ocorrência de cada espécie no total de unidades de amostra; a densidade mede a participação das diferentes espécies dentro da associação vegetal; a dominância permite medir o potencial de produção da floresta e constitui uma característica útil na determinação da qualidade de sítio (FINOL, 1971; LONGHI, 1980; LAMPRECHT, 1990); a distribuição diamétrica permite analisar o estado em que se encontra a floresta, assim como fazer inferências sobre a descontinuidade das classes diamétricas; as características ecofisiológicas das espécies e propor alternativas de manejo (SOUZA e JESUS, 1994); e a distribuição espacial permite determinar quais espécies e, ou, qual o número de indivíduos a serem explorados ou mantidos na área, de forma que a reprodução seja garantida (BARROS, 1980; ARAUJO et al., 2000).

A estrutura interna da floresta é outro aspecto de fundamental importância, sendo caracterizada pela qualidade e sanidade do fuste das árvores, especialmente as comercializáveis (CHICHORRO, 2000). Essas características fazem que as espécies sejam mais bem valoradas, havendo grande amplitude nos preços, desde espécies ainda desconhecidas no mercado (sem valor comercial) até espécies que atingem altos preços no mercado internacional.

Segundo Kelman (1975), para conhecer essas estruturas, necessita-se de uma base de dados fidedignos a serem analisados para gerar as estimativas dos parâmetros florísticos e fitossociológicos, bem como interpretações de critérios qualitativos e quantitativos, para que se possam realizar comparações entre os diferentes tipos de vegetação.

O conhecimento da composição florística e da estrutura da floresta permite o planejamento e estabelecimento de sistemas de manejo com produção 
sustentável, condução da floresta a uma estrutura balanceada, bem como práticas silviculturais adequadas. No sentido de viabilizar práticas de manejo em uma área de floresta ombrófila densa de terra firme, este estudo teve como objetivo analisar a composição florística e as estruturas horizontal, interna e diamétrica dessa floresta.

\section{MATERIAL E MÉTODOS}

O estudo foi realizado na unidade de manejo florestal (UMF) da Fazenda Tracajás (02³5’53”'S e 4747’10”W), empresa Nova Era Agroflorestal, de propriedade do Grupo Rosa Madeireira, Município de Paragominas, Estado do Pará, Brasil. A tipologia florestal é classificada como floresta ombrófila densa de terra firme em estádio de sucessão primária (clímax).

Na execução do plano de manejo florestal, destinado à produção de madeira para serraria e laminação, foi realizado o inventário de prospecção (100\%), no qual se estimaram os volumes do fuste comercial das árvores com dap $\geq 45$ cm de 55 espécies comerciais em 49 talhões de exploração de 10 hectares cada um, perfazendo uma área total de 490 hectares.

No inventário $100 \%$ das árvores comerciais com $d a p \geq 45 \mathrm{~cm}$ (diâmetro mínimo de corte), foram identificadas e marcadas as árvores-matriz, as árvores localizadas nas áreas de proteção ambiental e as árvores selecionadas para corte.

A unidade de manejo florestal constituída por 49 talhões de exploração foi estratificada em áreas homogêneas, com base no estoque de árvores comerciais das 55 espécies autorizadas para corte pelo IBAMA, doravante denominadas classes I, II e III de estoques volumétricos (SOUZA, 2003). Foram empregadas as análises de agrupamento (método de Ward) e discriminante (método de Fisher), na definição das classes de estoque. Na análise de agrupamentos, os talhões ou unidades de trabalho formaram grupos homogêneos e distintos, agrupados nas classes I, II e III de estoques volumétricos, com os respectivos volumes mínimo, médio e máximo e desvios-padrão (Quadro 1).

Em cada classe de estoque foram instaladas aleatoriamente cinco parcelas de 100 x 100 m (1 ha) cada uma, distribuídas de forma a cobrir todas as variações fisionômicas e estruturais da floresta (SILVA e LOPES, 1984).
Quadro 1 - Caracterização das classes de estoque volumétrico, mediante os valores dos volumes mínimo, médio, máximo e os desvios-padrão das árvores com dap $\geq 45 \mathrm{~cm}$ de 55 espécies comerciais

Table 1 - Characterization of the volumetric stock classes, by the value of standard deviation of maximum, average and minimum volumes of stems with $\mathrm{dbh}$ $\geq 45 \mathrm{~cm}$ of 55 commercial species

\begin{tabular}{ccccc}
\hline Classe de & \multicolumn{4}{c}{ Volume $\left(\mathrm{m}^{3} / \mathrm{ha}\right)$} \\
\cline { 2 - 5 } Estoque & Mínimo & Médio & Máximo & Desvio-Padrão \\
\hline I & 35,60 & 45,16 & 50,89 & 4,67 \\
II & 52,55 & 58,11 & 63,82 & 3,41 \\
III & 69,50 & 76,77 & 89,36 & 6,37 \\
\hline
\end{tabular}

A coleta dos dados de inventário florestal foi realizada em dois níveis de inclusão de dap. No nível I, foram medidos os indivíduos arbóreos dentro de cada parcela de 100 x 100 m (1 ha) com dap $\geq 15$ cm. No nível II, foi instalada no centro de cada parcela de $100 \times 100$ m (1 ha) uma subparcela de $10 \times 100$ m (0,1 ha), para medição dos indivíduos com $5 \mathrm{~cm} \leq$ dap $<15 \mathrm{~cm}$.

Nos níveis I e II de inclusão foram feitas as seguintes avaliações de cada árvore: nome vulgar regional e científico, medição do diâmetro a 1,30 m do solo (dap), altura comercial (Hc) e total (Ht), qualidade de fuste (QF), iluminação de copa (IC), cobertura de copa (CC), infestação de cipós (C) e danos naturais (Dn).

As espécies foram identificadas no campo, porém os indivíduos não identificados no local tiveram seus materiais botânicos coletados para identificação no Herbário do Museu Paraense Emílio Goeldi (MPEG), Estado do Pará.

No cálculo do número de parcelas, considerouse um erro de amostragem máximo de 20 a 95\% de probabilidade (IBAMA, 1998).

A composição florística foi analisada com base na distribuição dos indivíduos em espécies e famílias e no índice de Shannon-Weaver (BROWER e ZAR, 1984). Foram consideradas como espécies raras ou de baixa densidade aquelas que se apresentaram com até dois indivíduos por hectare (GOMES, 2000).

A classificação das espécies por grupo de uso e qualidade de fuste foi baseada em critérios estabelecidos por Yared et al. (1998) e Amaral et al. (1998), respectivamente.

Os parâmetros fitossociológicos da estrutura horizontal foram estimados, conforme Muller-Dombois

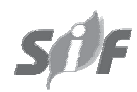

R. Árvore, Viçosa-MG, v.30, n.1, p.75-87, 2006 
e Ellemberg (1974). A estrutura diamétrica foi caracterizada por meio das distribuições do número de árvores, da área basal e do volume de fuste por hectare, por espécie e por classe de diâmetro. Para executar essa análise, as árvores com dap superior ou igual ao nível de inclusão de dap foram agrupadas em classes de dap, prefixandose a amplitude de classe de $10 \mathrm{~cm}$ (BARROS, 1980).

O volume de fuste com casca das árvores individuais foi estimado pelo emprego da equação $\hat{V}=0,0775+0,5179 \cdot d_{a p}^{2} \cdot H c(1)$, ajustada por Queiroz (1977), em que $\hat{V}=$ volume sem casca por árvore, em $\mathrm{m}^{3}$; dap = diâmetro à altura do peito com casca, em $\mathrm{cm}$; e Hc = altura comercial, em m.

O número de árvores por hectare, por espécie e por classe de diâmetro foi estimado pelo emprego da equação (MEYER, 1952) $Y_{j}=e^{\beta_{0}+\beta_{1} D_{j}}(2)$, sendo $Y_{j} o$ estimador do número de árvores por hectare na j-ésima classe de dap; $\mathrm{b}_{0}$ e $\mathrm{b}_{1}$, os coeficientes da equação; $\mathrm{D}_{\mathrm{j}}$, o diâmetro correspondente ao centro da j-ésima classe de dap; e e, a constante dos logarítmos neperianos.

Para comparar as distribuições das árvores (n/ ha), da área basal ( $\left.\mathrm{m}^{2} / \mathrm{ha}\right)$ e do volume $\left(\mathrm{m}^{3} / \mathrm{ha}\right)$, por espécie e por classe de dap, nas classes I, II e III de estoque, utilizou-se o teste $\mathrm{F}$, de Graybill $\left(\mathrm{FH}_{0}\right)$, a 5\% de probabilidade (GRAYBILL, 1976), com emprego da equação de regressão linear simples: $C_{i}=\beta_{0}+\beta_{1} C_{j}+\varepsilon$, em que $C_{i}=$ classe de estoque dependente); $C_{j}=$ classe de estoque independente; $\beta_{0}$ e $\beta_{1}=$ parâmetros do modelo; e $\varepsilon=$ erro aleatório. A hipótese testada foi:

$$
H_{0}:\left[\begin{array}{l}
\beta_{0} \\
\beta_{1}
\end{array}\right]=\left[\begin{array}{l}
0 \\
1
\end{array}\right] \text { “versus” } H_{a}: \text { não } H_{0}
$$

A não-rejeição de $\mathrm{H}_{0}$ indica que os vetores classes de estoque $C_{i}$ e $C_{j}$ são estatisticamente semelhantes.

\section{RESULTADOS E DISCUSSÃO}

\subsection{Composição Florística}

No nível I de inclusão ( $d a p \geq 15 \mathrm{~cm}$ ), verificouse maior riqueza de espécies (Quadro 2). Essa riqueza pode estar relacionada à capacidade de regenaração das espécies, distribuição dos indivíduos nos estratos arbóreos, ciclo de vida, associação entre espécies e capacidade produtiva do local.

Nos níveis I e II de inclusão (Quadro 2), destacaramse as classes I e III de estoques, por apresentarem valores de H' de 3,98 e 4,02, respectivamente, com H' comum de 3,97 e 4,18. Tais valores foram superiores aos encontrados em estudos realizados por Ribeiro et al. (1999), Maciel et al. (2000), Martins Pinto (2000) e Yared et al. (2000), em floresta tropical de terra firme, na Amazônia. Entretanto, as espécies com baixos valores de densidade absoluta na área inventariada contribuíram para os altos índices de diversidade de espécies na comunidade florestal.

Nas classes I, II e III de estoques volumétricos das árvores com dap $\geq 15 \mathrm{~cm}$ (nível I de inclusão), oito famílias botânicas contribuíram com um total de 260,8 (81,0\%), 251,6 (81,4\%) e 258,6 (82,4\%) indivíduos por hectare, respectivamente. $\mathrm{Na}$ classe I de estoque volumétrico das árvores com $5 \mathrm{~cm} \leq$ dap $<15 \mathrm{~cm}$ (nível II de inclusão), sete famílias representaram um total de 616 (73\%) indivíduos por hectare; nas classes II e III de estoque, oito famílias participaram com um total de 628 (73,5\%) e 692 (78,1\%) indivíduos por hectare, respectivamente. Resultados semelhantes foram evidenciados por Ribeiro et al. (1999), Barros et al. (2000), Maciel et al. (2000), Martins Pinto (2000), Yared (2000) e Lima Filho et al. (2001), em que poucas famílias botânicas apresentaram maior número de indivíduos por hectare, para florestas naturais tropicais de terra firme na Amazônia.

Quadro 2 - Estimativas de números de espécies (S), famílias (Fam) e indivíduos (N) e índices de Shannon-Weaver (H’), nas classes I, II e III de estoques volumétricos das árvores com dap $\geq 15 \mathrm{~cm}$ (nível I de inclusão) e com $5 \mathrm{~cm}$ $\leq$ dap $<15$ cm (nível II de inclusão), Fazenda Tracajás, Paragominas, Estado do Pará

Table 2 - Estimates of the number of species (S), family (Fam), individuals (N) and Shannon-Weaver (H') diversity index, per stock class I, II e III, level I and II inclusions; Tracajás Farm, Paragominas municipality, Pará State

\begin{tabular}{|c|c|c|c|c|c|c|c|c|}
\hline \multirow{2}{*}{$\begin{array}{l}\text { Classe de } \\
\text { Estoque }\end{array}$} & \multicolumn{4}{|c|}{ Nível I de Inclusão (dap $\geq 15 \mathrm{~cm}$ ) } & \multicolumn{4}{|c|}{ Nível II de Inclusão $(5 \mathrm{~cm} \leq$ dap $<15 \mathrm{~cm})$} \\
\hline & $\mathrm{S}$ & Fam & $\mathrm{N}$ & $\mathrm{H}^{\prime}$ & $\mathrm{S}$ & Fam & $\mathrm{N}$ & $\mathrm{H}^{\prime}$ \\
\hline I & 163 & 43 & 1.612 & 3,98 & 112 & 38 & 423 & 3,93 \\
\hline II & 153 & 41 & 1.545 & 3,80 & 103 & 38 & 427 & 3,85 \\
\hline III & 141 & 43 & 1.569 & 3,83 & 113 & 38 & 443 & 4,02 \\
\hline Total & 218 & 47 & 4.726 & 3,97 & 189 & 47 & 1.293 & 4,18 \\
\hline
\end{tabular}

R. Árvore, Viçosa-MG, v.30, n.1, p.75-87, 2006 


\subsection{Estrutura Horizontal}

Nas classes I, II e III de estoques volumétricos das árvores com dap $\geq 15 \mathrm{~cm}$ (nível I de inclusão), a dominância ( $\mathrm{m}^{2} / \mathrm{ha}$ ) e o volume de fuste ( $\mathrm{m}^{3} / \mathrm{ha}$ ) exibiram maiores valores (Quadro 3). Isso se deve ao número de indivíduos com maiores diâmetros e alturas. Apesar de as classes de estoques volumétricos das árvores com $5 \leq$ dap $<15$ cm (nível II de inclusão) apresentarem maior densidade absoluta (n/ha), os valores absolutos de dominância $\left(\mathrm{m}^{2} / \mathrm{ha}\right)$ e volume de fuste $\left(\mathrm{m}^{3} / \mathrm{ha}\right)$ foram baixos. Isso pode ser explicado pelo número de indivíduos com menores tamanhos (diâmetros e alturas).
Do número total de espécies identificadas nas classes I, II e III de estoques volumétricos das árvores com dap $\geq 15$ cm (nível I de inclusão), as espécies de maior valor de importância relativa (VI\%) contribuíram, respectivamente, com 49,0\%, 52,2\% e 50,8\% da densidade total (n/ha); 38,9\%, 45,3\% e 49,3\% da dominância total ( $\mathrm{m}^{2} / \mathrm{ha}$ ); 37,3\%, 45,2\% e 49,6\% do volume de fuste total ( $\mathrm{m}^{3} / \mathrm{ha}$ ); e 33,9\%, 37,3\% e 37,9\% do VI\% (Quadro 4).

Nas classes I, II e III de estoque, a espécie Lecythis idatimon (potencial)ocupou a primeira posição emimportância, graças aos elevados valores de densidade e dominância por hectare na área inventariada (Quadro 4).

Quadro 3 - Valores médios da densidade (n/ha), da dominância $\left(\mathrm{m}^{2} / \mathrm{ha}\right)$ e do volume de fuste $\left(\mathrm{m}^{3} / \mathrm{ha}\right)$ nas classes I, II e III de estoques volumétricos das árvores com dap $\geq 15 \mathrm{~cm}$ (nível I de inclusão) e com $5 \mathrm{~cm} \leq$ dap $<15 \mathrm{~cm}$ (nível II de inclusão), Fazenda Tracajás, Município de Paragominas, Estado do Pará

Table 3 - Average values of absolute density $(\mathrm{n} / \mathrm{ha})$, absolute dominance $\left(\mathrm{m}^{2} / \mathrm{ha}\right)$, whole-tree volume classes of the volumetric stocks I, II and III, level I and II inclusions, Tracajás Farm, Paragominas municipality, Pará State

\begin{tabular}{|c|c|c|c|c|c|c|}
\hline \multirow{2}{*}{$\begin{array}{l}\text { Classe de } \\
\text { Estoque } \\
\end{array}$} & \multicolumn{3}{|c|}{ Nível I de Inclusão (dap $\geq 15 \mathrm{~cm}$ ) } & \multicolumn{3}{|c|}{ Nível II de Inclusão $(5 \mathrm{~cm} \leq d a p<15 \mathrm{~cm})$} \\
\hline & $\mathrm{n} / \mathrm{ha}$ & $\mathrm{m}^{2} /$ há & $\mathrm{m}^{3} / \mathrm{ha}$ & $\mathrm{n} / \mathrm{ha}$ & $\mathrm{m}^{2} / \mathrm{ha}$ & $\mathrm{m}^{3} / \mathrm{ha}$ \\
\hline $\mathrm{I}$ & 322,4 & 27,36 & 358,69 & 846 & 4,80 & 93,98 \\
\hline II & 309,0 & 27,45 & 328,33 & 854 & 4,93 & 91,23 \\
\hline III & 313,8 & 25,88 & 308,69 & 886 & 5,46 & 97,61 \\
\hline Total & 315,0 & 26,90 & 331,90 & 862 & 5,06 & 94,27 \\
\hline
\end{tabular}

Quadro 4 - Estimativas dos parâmetros da estrutura horizontal da floresta ombrófila densa de terra firme não-explorada, por espécie, classes I, II e III de estoques volumétricos das árvores com dap ${ }^{3} 15$ cm (nível I de inclusão), Fazenda Tracajás, Paragominas, Estado do Pará

Table 4 - Estimates of the horizontal structure parameters in unexploited terra firme dense ombrophylous forest, per specie, volumetric stock classes I, II and III, level I inclusion, Tracajás Farm, Paragominas municipality, Pará State

\begin{tabular}{|c|c|c|c|c|c|c|c|c|c|c|c|c|}
\hline \multirow[t]{2}{*}{ Espécie } & \multicolumn{4}{|c|}{ Classe I de Estoque } & \multicolumn{4}{|c|}{ Classe II de Estoque } & \multicolumn{4}{|c|}{ Classe III de Estoque } \\
\hline & n/ha & $\mathrm{m}^{2} / \mathrm{ha}$ & $\mathrm{m}^{3} / \mathrm{ha}$ & VI(\%) & n/ha & $\mathrm{m}^{2} / \mathrm{ha}$ & $\mathrm{m}^{3} / \mathrm{ha}$ & VI(\%) & n/ha & $\mathrm{m}^{2} / \mathrm{ha}$ & $\mathrm{m}^{3} / \mathrm{ha}$ & VI(\%) \\
\hline Ecclinusa ramiflora Mart. & & & & & 6,6 & 0,55 & 6,58 & 1,9 & & & & \\
\hline $\begin{array}{l}\text { Eschweilera coriacea } \\
\text { Mart. ex O. Berg. }\end{array}$ & 20,6 & 1,88 & 23,57 & 4,9 & 28,6 & 2,87 & 33,88 & 7,1 & 31,8 & 2,64 & 29,995 & 7,2 \\
\hline Euxylophora paraensis Huber & & & & & & & & & 4,8 & 1,15 & 14,781 & 2,5 \\
\hline Lecythis idatimon Aubl. & 54,0 & 2,93 & 36,83 & 9,6 & 60,6 & 3,35 & 39,07 & 11,1 & 45,0 & 2,10 & 24,840 & 7,9 \\
\hline Licania canescens Benoist & 8,8 & 0,55 & 7,08 & 2,0 & 6,8 & 0,59 & 7,13 & 1,9 & 6,2 & 0,58 & 7,004 & 1,9 \\
\hline $\begin{array}{l}\text { Macrolobium acaciaefolium } \\
\text { Benth }\end{array}$ & 8,0 & 0,46 & 5,28 & 1,9 & & & & & 11,6 & 0,70 & 7,029 & 2,6 \\
\hline $\begin{array}{l}\text { Manilkara huberi Standl. } \\
\text { Micrandra elata Müll. Arg. }\end{array}$ & 6,0 & 0,51 & 6,60 & 1,7 & 4,6 & 0,83 & 11,21 & 2,0 & 4,2 & 0,76 & 9,741 & 1,9 \\
\hline $\begin{array}{l}\text { Pouteria decorticans } \\
\text { T. D. Penn. }\end{array}$ & 7,8 & 0,89 & 11,80 & 2,4 & 12,8 & 1,17 & 13,53 & 3,3 & 7,2 & 0,69 & 8,618 & 2,0 \\
\hline Pouteria gongrijpii Eyma & 6,6 & 0,49 & 6,09 & 1,7 & 8,8 & 0,47 & 5,41 & 2,0 & & & & \\
\hline Pouteria guianensis Aubl. & 9,0 & 0,80 & 10,41 & 2,4 & 10,6 & 1,20 & 14,68 & 3,1 & 10,0 & 0,76 & 8,938 & 2,5 \\
\hline Pouteria pachycarpa Pires & 8,6 & 0,66 & 8,44 & 2,2 & 8,4 & 0,62 & 7,21 & 2,1 & & & & \\
\hline $\begin{array}{l}\text { Rinorea guianensis Aubl. } \\
\text { Tachigali myrmecophila } \\
\text { Ducke }\end{array}$ & 28,6 & 1,47 & 17,66 & 5,2 & 13,4 & 0,80 & $\begin{array}{l}9,89 \\
7,35\end{array}$ & 2,9 & $\begin{array}{c}29,6 \\
5,6\end{array}$ & $\begin{array}{l}1,73 \\
1,31\end{array}$ & $\begin{array}{l}20,275 \\
16,770\end{array}$ & $\begin{array}{l}5,8 \\
2,7\end{array}$ \\
\hline$\overline{\text { Subtotal }}$ & 158,0 & 10,65 & 133,77 & 33,9 & 161,2 & 12,44 & 155,94 & 37,4 & 156,0 & 12,41 & 147,99 & 37,1 \\
\hline Demais espécies & 164,4 & 16,71 & 224,92 & 66,1 & 147,8 & 15,01 & 172,39 & 62,6 & 157,8 & 13,47 & 160,70 & 62,9 \\
\hline Total & 322,4 & 27,36 & 358,69 & 100,00 & 309 & 27,45 & 328,33 & 100,00 & 313,8 & 25,88 & 308,69 & 100,00 \\
\hline
\end{tabular}


Dentre as espécies de maior importância relativa (Quadro 4), Eschweilera coriacea (potencial), Lecythis idatimon (potencial), Licania canescens e Pouteria decorticans (potencial), Pouteria guianensis (potencial) e Rinorea guianensis (não-comercial) ocorreram em todas as classes de estoque, podendo ser consideradas importantes na composição florística da área inventariada.

Nas classes I, II e III de estoque, as espécies de maior importância Pouteria pachycarpa, Micrandra elata, Manilkara huberi, Tachigali myrmecophila e Euxylophora paraensis são comerciais (Quadro 4). Esses dados indicam que a maioria das espécies comerciais apresenta baixos valores absolutos de densidade por hectare na área inventariada.

Do número total de espécies indentificadas nas classes I, II e III de estoques volumétricos das árvores com $5 \mathrm{~cm} \leq$ dap $<15 \mathrm{~cm}$ (nível II de inclusão), as espécies de maior valor de importância relativa contribuíram, respectivamente, com 47,3\%, 49,9\% e 43,6\% da densidade total (n/ha); 48,3\%, 49,3\% e 42,3\% da dominância total ( $\left.\mathrm{m}^{2} / \mathrm{ha}\right) ; 47,1 \%, 50,6 \%$ e 43,7\% do volume de fuste total ( $\mathrm{m}^{3} / \mathrm{ha}$ ); e 39,3\%, 41,2\% e 36,6\% do VI\% (Quadro 5).

Nas classes I, II e III de estoque, as espécies Lecythis idatimon (potencial), Dodecastigma integrifolium (nãocomercial) e Rinorea guianensis (não-comercial) ocuparam o primeiro lugar em importância devido aos elevados valores absolutos de densidade e dominância por hectare na área inventariada (Quadro 5).

Dentre as espécies de maior importância relativa (Quadro 5), Lecythis idatimon (potencial), Dodecastigma integrifolium (não-comercial), Rinorea guianensis (nãocomercial) e Pouteria guianensis (potencial) ocorreram em todas as classes de estoque, sendo consideradas importantes na composição florística da área inventariada.

Quadro 5 - Estimativas dos parâmetros da estrutura horizontal da floresta ombrófila densa de terra firme não explorada, por espécie, classes I, II e III de estoques volumétricos das árvores com $5 \mathrm{~cm} \leq d a p<15 \mathrm{~cm}$ (nível II de inclusão), Fazenda Tracajás, Paragominas, Estado do Pará

Table 5 - Estimates of the horizontal structure parameters in unexploited terra firme dense ombrophylous forest, per specie, volumetric stock classes I, II and III, inclusion level II, Tracajás Farm, Paragominas municipality, Pará State

\begin{tabular}{|c|c|c|c|c|c|c|c|c|c|c|c|c|}
\hline \multirow[t]{2}{*}{ Espécie } & \multicolumn{4}{|c|}{ Classe I de Estoque } & \multicolumn{4}{|c|}{ Classe II de Estoque } & \multicolumn{4}{|c|}{ Classe III de Estoque } \\
\hline & $\mathrm{n} / \mathrm{ha}$ & $\mathrm{m}^{2} / \mathrm{ha}$ & $\mathrm{m}^{3} / \mathrm{ha}$ & VI(\%) & $\mathrm{n} / \mathrm{ha}$ & $\mathrm{m}^{2} / \mathrm{ha}$ & $\mathrm{m}^{3} / \mathrm{ha}$ & VI(\%) & $\mathrm{n} / \mathrm{ha}$ & $\mathrm{m}^{2} / \mathrm{ha}$ & $\mathrm{m}^{3} / \mathrm{ha}$ & $\overline{\mathrm{VI}(\%)}$ \\
\hline $\begin{array}{l}\text { Dodecastigma integrifolium } \\
\text { Lanj. \& Sandwith }\end{array}$ & 94 & 0,53 & 9,67 & 8,3 & 108 & 0,63 & 11,41 & 9,2 & 76 & 0,39 & 7,25 & 6,0 \\
\hline $\begin{array}{l}\text { Eschweilera coriacea } \\
\text { Mart. ex O. Berg }\end{array}$ & 18 & 0,11 & 2,07 & 2,2 & & & & & & & & \\
\hline $\begin{array}{l}\text { Eschweilera pedicellata } \\
\text { (Rich.) S.A. Mori }\end{array}$ & 14 & 0,10 & 1,74 & 1,9 & & & & & 26 & 0,16 & 2,75 & 2,6 \\
\hline Inga lateriflora Miq. & & & & & 14 & 0,10 & 1,59 & 1,7 & & & & \\
\hline Inga nobilis Willd. & 12 & 0,06 & 1,22 & 1,6 & 22 & 0,14 & 2,39 & 2,5 & & & & \\
\hline Lecythis idatimon Aubl. & 98 & 0,64 & 11,89 & 9,2 & 86 & 0,47 & 9,29 & 7,4 & 78 & 0,59 & 9,56 & 7,3 \\
\hline $\begin{array}{l}\text { Macrolobium acaciaefolium } \\
\text { Benth. }\end{array}$ & & & & & & & & & 20 & 0,15 & 2,35 & 2,0 \\
\hline Micropholis guyanensis Pierre & 14 & 0,09 & 1,66 & 1,7 & & & & & & & & \\
\hline Peltogyne leicointei Ducke & & & & & 14 & 0,09 & 1,51 & 1,7 & & & & \\
\hline Poecilanthe effusa Ducke & 22 & 0,11 & 2,19 & 2,3 & & & & & & & & \\
\hline Pouteria gongrijpii Eyma & & & & & & & & & 24 & 0,11 & 2,59 & 2,2 \\
\hline Pouteria guianensis Aubl. & 12 & 0,06 & 1,32 & 1,6 & 50 & 0,27 & 5,35 & 4,7 & 32 & 0,22 & 3,72 & 3,4 \\
\hline Pouteria pachycarpa Pires & & & & & & & & & 18 & 0,11 & 2,05 & 1,8 \\
\hline $\begin{array}{l}\text { Protium aracouchili } \\
\text { (Aubl.) March. }\end{array}$ & 40 & 0,20 & 4,38 & 3,8 & & & & & 14 & 0,12 & 1,87 & 1,9 \\
\hline $\begin{array}{l}\text { Protium heptaphyllum } \\
\text { (Aubl.) March. }\end{array}$ & & & & & 16 & 0,10 & 1,79 & 1,9 & & & & \\
\hline $\begin{array}{l}\text { Protium spruceanum } \\
\text { (Benth.) Engl. }\end{array}$ & & & & & 20 & 0,12 & 2,25 & 2,4 & 24 & 0,13 & 2,67 & 2,2 \\
\hline Rinorea guianensis Aubl. & 76 & 0,42 & 8,14 & 6,6 & 78 & 0,51 & 8,65 & 7,4 & 94 & 0,56 & 10,08 & 7,7 \\
\hline Sterculia speciosa K. Schum & & & & & 18 & 0,10 & 1,98 & 2,1 & & & & \\
\hline Subtotal & 400 & 2,31 & 44,29 & 39,3 & 426 & 2,54 & 46,22 & 41,2 & 406 & 2,53 & 44,89 & 37,2 \\
\hline Demais espécies & 446 & 2,49 & 49,69 & 60,7 & 428 & 2,40 & 45,02 & 58,8 & 480 & 2,93 & 52,72 & 62,8 \\
\hline Total & 846 & 4,80 & 93,98 & 100,0 & 854 & 4,93 & 91,24 & 100,0 & 886 & 5,46 & 97,61 & $\overline{100,0}$ \\
\hline
\end{tabular}

R. Árvore, Viçosa-MG, v.30, n.1, p.75-87, 2006 
Nas classes I, II e III de estoque (Quadro 5), as espécies de maior valor de importância Protium aracouchili, Micropholis guyanensis, Protium spruceanum, Sterculia speciosa, Protium heptaphyllum, Peltogyne leicointei e Pouteria pachycarpa são comerciais.

Lecythis idatimon (potencial), Rinorea guianensis (não-comercial) e Pouteria guianensis (potencial) ocorreram em todos os níveis de inclusão e classes de estoque. A grande maioria das espécies ocorreu em baixas densidades por hectare, o que confere a essas espécies menor importância na vegetação.

Conhecer a estrutura populacional em termos de densidade absoluta ( $\mathrm{n} / \mathrm{ha}$ ) permite estabelecer sistema de manejo com produção sustentável, bem como tratamentos silviculturais para estimular e regularizar a regeneração das comerciais e potenciais raras e de baixa densidade absoluta.

Nos níveis I e II de inclusão, Myracrodruon urundeuva Allemão, Bowdichia nitida Spruce ex Benth e Euxylophora paraensis constam na lista de espécies da flora brasileira ameaçadas de extinção (IBAMA, 1992). Além dessas, Batesia floribunda Spruce ex Benth, Brosimum parinarioides Ducke, Brosimum rubescens Taub., Buchenavia parvifolia Ducke, Minquartia guianensis Aubl., Ocotea rubra Mez, Parkia pendula Benth. Ex Walp. e Sacoglottis amazonica Mart. foram classificadas como espécies potencialmente ameaçadas pela exploração madeireira na Amazônia (AMARAL et al., 1998). Por conseguinte, as referidas espécies são imunes de corte ou de aproveitamento econômico na área inventariada.

Os valores médios da densidade (n/ha), da dominância $\left(\mathrm{m}^{2} / \mathrm{ha}\right.$ ) e do volume de fuste ( $\mathrm{m}^{3} / \mathrm{ha}$ ) nos grupos de usos entre as classes I, II e III de estoques volumétricos (nível I de inclusão) encontram-se no Quadro 6.

Em termos de valores médios de densidade absoluta, destacaram-se as espécies potenciais (Quadro 6), porém o grupo de espécies comerciais apresentou maior área basal e volume. Esse fato pode ser atribuído ao número de indivíduos de espécies comerciais com maiores diâmetros e alturas presentes na floresta.

O predomínio do grupo de espécies potenciais em densidade de indivíduos (Quadro 6) confere a perspectiva de ampliação do valor econômico da floresta, pelo fato de essas espécies apresentarem características tecnológicas e de uso que possibilitam seu comércio futuro. Em vista disso, os tratamentos silviculturais devem contemplar tanto as espécies comerciais quanto as potenciais para garantir a produção sustentável de produtos florestais madeireiros.

Os valores médios da densidade (n/ha), da dominância $\left(\mathrm{m}^{2} / \mathrm{ha}\right)$ e do volume de fuste $\left(\mathrm{m}^{3} / \mathrm{ha}\right)$ para os grupos de usos entre as classes I, II e III de estoques volumétricos (nível II de inclusão) encontram-se no Quadro 7.

Quadro 6 - Valores médios da densidade absoluta (n/ha), da dominância absoluta ( $\mathrm{m}^{2} / \mathrm{ha}$ ) e do volume de fuste ( $\left.\mathrm{m}^{3} / \mathrm{ha}\right)$ nos grupos de usos entre as classes I, II e III de estoques volumétricos, nível I de inclusão, Fazenda Tracajás, Paragominas, Estado do Pará

Table 6 - Average values of absolute density ( $\mathrm{n} / \mathrm{ha})$, absolute dominance $\left(\mathrm{m}^{2} / \mathrm{ha}\right)$, whole-tree volume for groups of use among volumetric stock classes I, II and III, level I inclusion, Tracajás Farm, Paragominas, Pará State

\begin{tabular}{|c|c|c|c|c|c|c|c|c|c|}
\hline \multirow[t]{2}{*}{ Classe de Estoque } & \multicolumn{3}{|c|}{ Comercial } & \multicolumn{3}{|c|}{ Potencial } & \multicolumn{3}{|c|}{ Não-Comercial } \\
\hline & $\mathrm{n} / \mathrm{ha}$ & $\mathrm{m}^{2} / \mathrm{ha}$ & $\mathrm{m}^{3} / \mathrm{ha}$ & $\mathrm{n} / \mathrm{ha}$ & $\mathrm{m}^{2} / \mathrm{ha}$ & $\mathrm{m}^{3} / \mathrm{ha}$ & n/ha & $\mathrm{m}^{2} / \mathrm{ha}$ & $\mathrm{m}^{3} / \mathrm{ha}$ \\
\hline $\mathrm{I}$ & 117,8 & 13,34 & 182,17 & 156,4 & 11,47 & 145,89 & 48,2 & 2,55 & 30,63 \\
\hline II & 106,6 & 12,94 & 156,80 & 167,2 & 12,50 & 147,44 & 35,2 & 2,01 & 24,09 \\
\hline III & 110,4 & 12,65 & 156,01 & 153,8 & 10,37 & 120,33 & 49,6 & 2,85 & 32,35 \\
\hline
\end{tabular}

Quadro 7 - Valores médios da densidade absoluta (n/ha), da dominância absoluta ( $\mathrm{m}^{2} / \mathrm{ha}$ ) e do volume de fuste (m³/ha) nos grupos de usos entre as classes I, II e III de estoques volumétricos, nível II de inclusão, Fazenda Tracajás, Paragominas, Estado do Pará

Table 7 - Average values of absolute density ( $\mathrm{n} / \mathrm{ha})$, absolute dominance ( $\left.\mathrm{m}^{2} / \mathrm{ha}\right)$, whole-tree volume for groups of use among volumetric stock classes I, II and III, level II inclusion, Tracajás Farm, Paragominas, Pará State

\begin{tabular}{cccccccccc}
\hline Classe de Estoque & \multicolumn{3}{c}{ Comercial } & \multicolumn{3}{c}{ Potencial } & \multicolumn{3}{c}{ Não-Comercial } \\
\cline { 2 - 8 } & $\mathrm{n} / \mathrm{ha}$ & $\mathrm{m}^{2} / \mathrm{ha}$ & $\mathrm{m}^{3} / \mathrm{ha}$ & $\mathrm{n} / \mathrm{ha}$ & $\mathrm{m}^{2} / \mathrm{ha}$ & $\mathrm{m}^{3} / \mathrm{ha}$ & $\mathrm{n} / \mathrm{ha}$ & $\mathrm{m}^{2} / \mathrm{ha}^{3} / \mathrm{m}^{3}$ \\
\hline I & 224 & 1,25 & 25,16 & 230 & 1,48 & 27,35 & 392 & 2,07 & 41,47 \\
II & 206 & 1,24 & 22,54 & 230 & 1,31 & 24,96 & 418 & 2,38 & 43,73 \\
III & 242 & 1,54 & 27,63 & 254 & 1,63 & 28,76 & 390 & 2,29 & 41,22 \\
\hline
\end{tabular}


Em termos de valores médios, a densidade, a dominância e o volume de fuste das espécies nãocomerciais foram superiores aos das comerciais (Quadro 7). Esses dados indicam que os tratamentos silviculturais devem ser aplicados para regular os estoques das espécies comerciais e, sobretudo, promover as espécies não-comerciais no mercado.

\subsection{Estrutura Interna}

Os valores médios da densidade (n/ha), dominância $\left(\mathrm{m}^{2} / \mathrm{ha}\right)$ e do volume de fuste $\left(\mathrm{m}^{3} / \mathrm{ha}\right.$ ) para as classes de fuste entre as classes I, II e III de estoques volumétricos (nível I de inclusão) encontram-se no Quadro 8.

Nas classes I, II e III de estoques volumétricos, verificou-se que as árvores com fustes bons representaram 274,0 (85,0\%) árvores/ha, 279,2 (90,4\%) árvores/ha e 296,2 (94,4\%) árvores/ha, respectivamente (Quadro 8). Desse total, as árvores com valor comercial futuro participaram, respectivamente, com 142,4(52,0\%)árvores/ha, 155,4(55,6\%) árvores/ha e 146,8 (49,6\%) árvores/ha. No entanto, as árvores de valor comercial representaram 92 (33,6\%)árvores/ ha, 93 (33,3\%) árvores/ha e 104,6 (35,3\%) árvores/ha, respectivamente. Esses dados indicam que os tratamentos silviculturais devem beneficiar tanto as espécies comerciais quanto as potenciais para melhorar a qualidade e a produtividade da floresta e, sobretudo, promover o crescimento e a produção de árvores de valor comercial.

Os valores médios da densidade (n/ha), dominância ( $\mathrm{m}^{2} / \mathrm{ha}$ ) e do volume de fuste $\left(\mathrm{m}^{3} / \mathrm{ha}\right)$ para as classes de fuste entre as classes I, II e III de estoques volumétricos (nível II de inclusão) constam no Quadro 9.

Verificou-se, nas classes I, II e III de estoques volumétricos, que as árvores com fustes bons participaram com 532 (63,0\%) árvores/ha, 556 (65,0\%) árvores/ha e 580 (65,0\%) árvores/ha, respectivamente (Quadro 9). Desse total, as árvores com fustes de boa qualidade, 228 (43,0\%) árvores/ha, 282 (51,0\%) árvores/ ha e 232 (40,0\%) árvores/ha, respectivamente, são de espécies não-comerciais. Esses dados indicam que tanto as espécies comerciais quanto as potenciais devem receber tratamentos silviculturais para promover o crescimento e a produção de árvores de maior valor econômico, bem como sustentar a produção de produtos florestais madeireiros.

Quadro 8 - Valores médios da densidade absoluta (n/ha), da dominância absoluta ( $\mathrm{m}^{2} / \mathrm{ha}$ ) e do volume de fuste ( $\mathrm{m}^{3} / \mathrm{ha}$ ) nas classes de fuste entre as classes I, II e III de estoques volumétricos, nível I de inclusão, Fazenda Tracajás, Paragominas, Estado do Pará

Table 8 -Average values of absolute density $(\mathrm{n} / \mathrm{ha})$, absolute dominance $\left(\mathrm{m}^{2} / \mathrm{ha}\right)$, whole-tree volume for whole-tree classes among volumetric stock classes I, II and III, level I inclusion, Tracajás Farm, Paragominas municipality, Pará State

\begin{tabular}{|c|c|c|c|c|c|c|c|c|c|}
\hline \multirow[t]{3}{*}{ Classe de Estoque } & \multicolumn{3}{|c|}{ Fuste Bom } & \multicolumn{3}{|c|}{ Fuste Regular } & \multicolumn{3}{|c|}{ Fuste Inferior } \\
\hline & \multicolumn{3}{|c|}{ (80 a $100 \%$ de Aproveitamento) } & \multicolumn{3}{|c|}{ (50 a $79 \%$ de Aproveitamento) } & \multicolumn{3}{|c|}{ ( $<50 \%$ de Aproveitamento) } \\
\hline & n/ha & $\mathrm{m}^{2} / \mathrm{ha}$ & $\mathrm{M}^{3} / \mathrm{ha}$ & n/ha & $\mathrm{m}^{2} / \mathrm{ha}$ & $\mathrm{m}^{3} / \mathrm{ha}$ & n/ha & $\mathrm{m}^{2} / \mathrm{ha}$ & $\mathrm{m}^{3} / \mathrm{ha}$ \\
\hline $\mathrm{I}$ & 274,0 & 24,18 & 312,79 & 41,6 & 2,67 & 31,31 & 6,8 & 0,50 & 5,59 \\
\hline II & 279,2 & 25,19 & 304,10 & 25,6 & 2,00 & 21,76 & 4,2 & 0,27 & 2,45 \\
\hline III & 296,2 & 24,60 & 296,00 & 14,6 & 1,05 & 10,88 & 3,0 & 0,19 & 1,78 \\
\hline
\end{tabular}

Quadro 9 - Valores médios da densidade absoluta (n/ha), da dominância absoluta ( $\mathrm{m}^{2} / \mathrm{ha}$ ) e do volume de fuste ( $\left.\mathrm{m}^{3} / \mathrm{ha}\right)$ nas classes de fuste entre as classes I, II e III de estoques volumétricos, nível II de inclusão, Fazenda Tracajás, Paragominas, Pará

Table 9 - Average values of absolute density $(\mathrm{n} / \mathrm{ha})$, absolute dominance $\left(\mathrm{m}^{2} / \mathrm{ha}\right)$, whole-tree volume for whole-tree classes among volumetric stock classes I, II and III, level II inclusion, Tracajás Farm, Paragominas municipality, Pará State

\begin{tabular}{|c|c|c|c|c|c|c|c|c|c|}
\hline \multirow[t]{3}{*}{ Classe de Estoque } & \multicolumn{3}{|c|}{ Fuste Bom } & \multicolumn{3}{|c|}{ Fuste Regular } & \multirow{2}{*}{\multicolumn{3}{|c|}{$\begin{array}{c}\text { Fuste Inferior } \\
(<50 \% \text { de Aproveitamento })\end{array}$}} \\
\hline & \multicolumn{3}{|c|}{ (80 a $100 \%$ de Aproveitamento) } & \multirow{2}{*}{$\frac{(50 \text { a } 79}{n / h a}$} & \multicolumn{2}{|c|}{ \% de Aproveitamento) } & & & \\
\hline & n/ha & $\mathrm{m}^{2} / \mathrm{ha}$ & $\mathrm{M}^{3} / \mathrm{ha}$ & & $\mathrm{m}^{2} / \mathrm{ha}$ & $\mathrm{m}^{3} / \mathrm{ha}$ & n/ha & $\mathrm{m}^{2} / \mathrm{ha}$ & $\mathrm{m}^{3} / \mathrm{ha}$ \\
\hline $\mathrm{I}$ & 532 & 3,12 & 60,80 & 246 & 1,31 & 26,26 & 68 & 0,37 & 6,85 \\
\hline II & 556 & 3,30 & 60,25 & 236 & 1,26 & 24,52 & 62 & 0,38 & 6,46 \\
\hline III & 580 & 3,63 & 64,85 & 250 & 1,55 & 27,41 & 56 & 0,28 & 5,35 \\
\hline
\end{tabular}




\subsection{Estrutura Diamétrica}

A distribuição dos números de árvores por hectare, por espécie e por classe de diâmetro, com amplitude de $10 \mathrm{~cm}$ das classes I, II e III de estoque (Figura 1), seguiu a distribuição em “J-invertido”, que é típica das florestas naturais ineqüiâneas.

As distribuições de diâmetros de todas as espécies e das espécies comerciais com dap $\geq 5 \mathrm{~cm}$ estimadas pela função de Meyer, representada pela expressão (2), confirmaram a tendência exponencial negativa (Quadro 10).

Nas classes I, II e III de estoque, agrupamento das árvores (n/ha), área basal ( $\mathrm{m}^{2} / \mathrm{ha}$ ) e volume de fuste
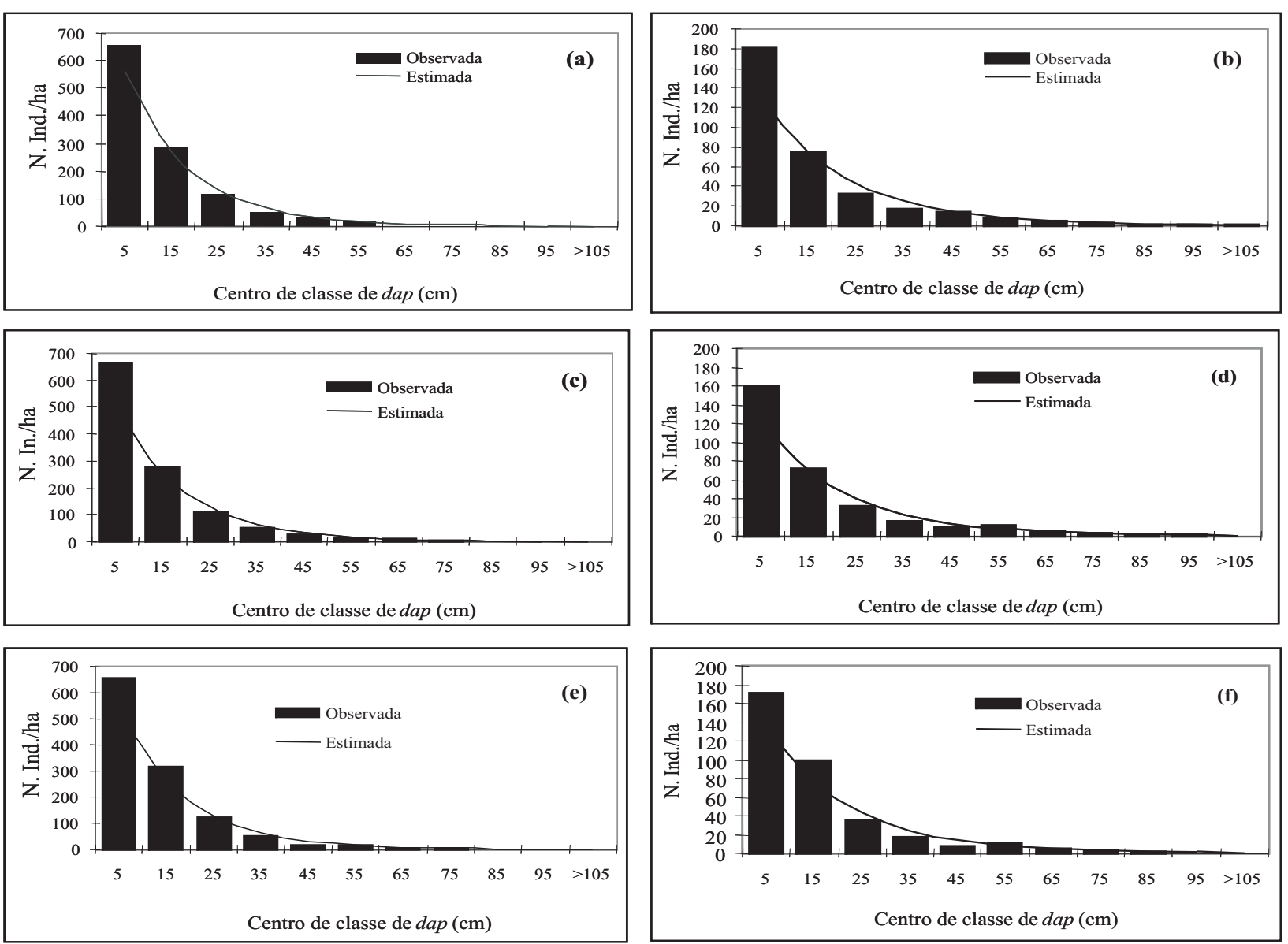

( $\left.\mathrm{m}^{3} / \mathrm{ha}\right)$ em classes de diâmetro com intervalo de 10 cm encontram-se nos Quadros 11 e 12.

Na classe III de estoque, observou-se maior valor absoluto da densidade total (n/ha), porém valores absolutos da dominância total ( $\mathrm{m}^{2} / \mathrm{ha}$ ) e do volume de fuste total $\left(\mathrm{m}^{3} / \mathrm{ha}\right.$ ) mais baixos. Isso pode ser explicado pelo número de indivíduos com menores tamanhos (diâmetro e altura) na classe de dap $\geq 45 \mathrm{~cm}$ (Quadro 11).

A estrutura diamétrica da floresta caracteriza-se por árvores de pequeno porte nas menores classes de diâmetro, indicando tendência de distribuição balanceada (Quadro 11). Isso se deve á capacidade de regeneração das espécies vegetais.

Figura 1 - Distribuição dos números de árvores por classe de diâmetro de todas as espécies (a, c, e) e das espécies comerciais (b, d, f), respectivamente, nas classes I, II e III de estoque, Fazenda Tracajás, Paragominas, Estado do Pará.

Figure 1 -Distribution of the number of trees per diameter class of all the species ( $a, c$ and $e$ ) and of the commercial especies( $b$, $d$ and $f$ ), respectively, for the classes of stock I, II and III, Tracajás Farm, Paragominas municipality, Pará state. 
Quadro 10 - Coeficientes e parâmetros estatísticos obtidos pela equação de Meyer Table 10 - Coefficient and statistic parameters obtained by the Meyer's equation

\begin{tabular}{ccccccccc}
\hline Classe de Estoque & \multicolumn{4}{c}{ Todas as Espécies } & \multicolumn{3}{c}{ Espécies Comerciais } \\
\cline { 2 - 9 } & $\hat{\beta}_{0}$ & $\hat{\beta}_{1}$ & $\bar{R}^{2}$ & $\mathrm{~S}_{\mathrm{yx}}$ & $\hat{\beta}_{0}$ & $\hat{\beta}_{1}$ & $\bar{R}^{2}$ & $\mathrm{~S}_{\mathrm{yx}}$ \\
\hline I & 6,698 & $-0,073$ & 0,99 & 0,18 & 5,116 & $-0,056$ & 0,98 & 0,21 \\
II & 6,566 & $-0,069$ & 0,98 & 0,26 & 5,081 & $-0,055$ & 0,96 & 0,28 \\
III & 6,630 & $-0,071$ & 0,98 & 0,26 & 5,195 & $-0,057$ & 0,95 & 0,34 \\
\hline
\end{tabular}

Quadro 11 - Agrupamento das árvores (n/ha), área basal ( $\left.\mathrm{m}^{2} / \mathrm{ha}\right)$ e volume de fuste $\left(\mathrm{m}^{3} / \mathrm{ha}\right)$ por classe de diâmetro de todas as espécies, nas classes I, II e III de estoque, Fazenda Tracajás, Paragominas, Estado do Pará

Table 11 - Cluster of trees stems $(\mathrm{n} / \mathrm{ha})$, basal area $\left(\mathrm{m}^{2} / \mathrm{ha}\right)$ and whole tree volume $\left(\mathrm{m}^{3} / \mathrm{ha}\right)$ per diameter of class for all the species, in the classes of stock I, II and III, Tracajás Farm, Paragominas municipality, Pará State

\begin{tabular}{cccccccccr}
\hline Classe de dap $(\mathrm{cm})$ & \multicolumn{3}{c}{ Classe I de Estoque } & \multicolumn{3}{c}{ Classe II de Estoque } & \multicolumn{3}{c}{ Classe III de Estoque } \\
\cline { 2 - 9 } & $\mathrm{n} / \mathrm{ha}$ & $\mathrm{m}^{2} / \mathrm{ha}$ & $\mathrm{m}^{3} / \mathrm{ha}$ & $\mathrm{n} / \mathrm{ha}$ & $\mathrm{m}^{2} / \mathrm{ha}$ & $\mathrm{m}^{3} / \mathrm{ha}$ & $\mathrm{n} / \mathrm{ha}$ & $\mathrm{m}^{2} / \mathrm{ha}$ & $\mathrm{m}^{3} / \mathrm{ha}$ \\
\hline $5-15$ & 658,0 & 2,73 & 65,05 & 666,0 & 2,81 & 63,60 & 660,0 & 2,83 & 63,67 \\
$15-35$ & 399,0 & 9,72 & 121,65 & 386,0 & 9,45 & 110,14 & 438,0 & 10,47 & 121,47 \\
$35-45$ & 49,0 & 4,48 & 55,77 & 51,0 & 4,68 & 53,69 & 51,0 & 4,69 & 52,77 \\
\hline$\geq 45$ & 63,0 & 15,24 & 210,18 & 60,0 & 15,44 & 192,15 & 51,0 & 13,35 & 168,40 \\
\hline Total & $1.169,0$ & 32,17 & 452,65 & $1.163,0$ & 32,38 & 419,58 & $1.200,0$ & 31,34 & 406,31 \\
\hline
\end{tabular}

Quadro 12 - Agrupamento das árvores (n/ha), área basal ( $\left.\mathrm{m}^{2} / \mathrm{ha}\right)$ e volume de fuste ( $\mathrm{m}^{3} / \mathrm{ha}$ ) por classe de diâmetro das espécies comerciais, nas classes I, II e III de estoque, Fazenda Tracajás, Paragominas, Estado do Pará

Table 12 - Cluster of trees $(\mathrm{n} / \mathrm{ha})$, basal area $\left(\mathrm{m}^{2} / \mathrm{ha}\right)$ and whole tree volume $\left(\mathrm{m}^{3} / \mathrm{ha}\right)$ per diameter of class of commercial species, in classes of stock I, II and III, Tracajás Farm, Paragominas municipality, Pará State

\begin{tabular}{cccccccccr}
\hline Classe de dap $(\mathrm{cm})$ & \multicolumn{3}{c}{ Classe I de Estoque } & \multicolumn{3}{c}{ Classe II de Estoque } & \multicolumn{3}{c}{ Classe III de Estoque } \\
\cline { 2 - 9 } & $\mathrm{n} / \mathrm{ha}$ & $\mathrm{m}^{2} / \mathrm{ha}$ & $\mathrm{m}^{3} / \mathrm{ha}$ & $\mathrm{n} / \mathrm{ha}$ & $\mathrm{m}^{2} / \mathrm{ha}$ & $\mathrm{m}^{3} / \mathrm{ha}$ & $\mathrm{n} / \mathrm{ha}$ & $\mathrm{m}^{2} / \mathrm{ha}$ & $\mathrm{m}^{3} / \mathrm{ha}$ \\
\hline $5-15$ & 182,0 & 0,79 & 18,48 & 160,0 & 0,71 & 15,59 & 172,0 & 0,74 & 16,91 \\
$15-35$ & 109,0 & 2,82 & 33,52 & 104,0 & 2,66 & 29,75 & 133,0 & 3,01 & 36,11 \\
$35-45$ & 17,8 & 1,66 & 21,28 & 16,2 & 1,53 & 17,88 & 17,8 & 1,66 & 18,75 \\
\hline$\geq 45$ & 33,0 & 9,33 & 132,01 & 32,4 & 9,28 & 105,32 & 30,0 & 8,80 & 112,52 \\
\hline Total & 341,8 & 14,60 & 205,29 & 312,6 & 14,18 & 168,54 & 352,8 & 14,21 & 184,29 \\
\hline
\end{tabular}

Nas classes I, II e III de estoque, as espécies comerciais participaram, respectivamente, com $29,2 \%$, 26,9\% e 29,4\% da densidade total (n/ha); 45,4\%, 43,8\% e $45,3 \%$ da dominância total ( $\mathrm{m}^{2} / \mathrm{ha}$ ); e $45,4 \%, 40,2 \%$ e $45,4 \%$ do volume de fuste total ( $\left.\mathrm{m}^{3} / \mathrm{ha}\right)$, de todas as espécies (Quadro 12), sendo os daps máximos de 127; 118,0; e 131,0 cm, referentes às espécies comerciais Goupia glabra (Gmel.) Aubl., Caryocar villosum (Aubl.) Pers.e Ocotea rubra Mez.

Segundo Souza e Jesus (1994), a distribuição de diâmetros permite analisar o estado em que se encontra a floresta, bem como fazer inferências sobre a descontinuidade das classes diamétricas, as características ecofisiológicas das espécies e propor alternativas de manejo. Portanto, a análise da estrutura diamétrica serve como critério de avaliação da sustentabilidade do manejo de florestas ineqüiâneas.
Considerando as árvores comerciais com dap $\geq$ $45 \mathrm{~cm}$ (diâmetro mínimo de corte) e volume de corte permissível de no máximo $35 \mathrm{~m}^{3} / \mathrm{ha}$, a colheita sob manejo florestal não compromete a capacidade de renovação e sustentação do estoque comercial remanescente nas classes I, II e III de estoque (Quadro 12).

As comparações feitas para as variáveis número de árvores, área basal e volume comercial por hectare, por espécie e por classe de diâmetro entre as classes de estoque I, II e III (Quadro 13), apontaram efeito não-significativo $(\mathrm{P}>0,05)$ pelo teste $\mathrm{F}$ de Graybill, exceto para número de árvores das espécies comerciais entre as classes de estoques I x II e II x III. Essa diferença $(\mathrm{P}<0,05)$ pode ser explicada pelo menor número de indivíduos por hectare estimado nas classe de dap entre 5 e $15 \mathrm{~cm}, 15$ e $35 \mathrm{~cm}$ (classe II de estoque), comparada com as demais classes de estoque. 
Quadro 13 - Comparações das distribuições do número de árvores (n/ha), da área basal ( $\mathrm{m}^{2} / \mathrm{ha}$ ) e do volume de fuste ( $\mathrm{m}^{3} /$ ha), pelo teste F de Graybill (5\%), entre as classes I, II e III de estoque, em todas as espécies e nas espécies comerciais

Table 13 - Comparison of the distribution of number of trees ( $\mathrm{n} / \mathrm{ha})$, basal area $\left(\mathrm{m}^{2} / \mathrm{ha}\right)$, and volume of the whole-tree $\left(\mathrm{m}^{3} / \mathrm{ha}\right)$, by the Graybill's F test (5\%), among the stock classes I, II e III, for all the species and commercial species

\begin{tabular}{|c|c|c|c|c|c|c|}
\hline \multirow[t]{2}{*}{ Classe de Estoque } & \multicolumn{3}{|c|}{ Todas as Espécies } & \multicolumn{3}{|c|}{ Espécies Comerciais } \\
\hline & $\mathrm{n} / \mathrm{ha}$ & $\mathrm{m}^{2} / \mathrm{ha}$ & $\mathrm{m}^{3} / \mathrm{ha}$ & n/ha & $\mathrm{m}^{2} / \mathrm{ha}$ & $\mathrm{m}^{3} /$ ha \\
\hline I $\mathrm{x}$ II & $0,372^{\text {ns }}$ & $0,754^{\mathrm{ns}}$ & $2,368^{\mathrm{ns}}$ & $23,545 *$ & $0,134^{\mathrm{ns}}$ & $1,133^{\mathrm{ns}}$ \\
\hline I $x$ III & $1,004^{\mathrm{ns}}$ & $0,197^{\mathrm{ns}}$ & $1,634^{\mathrm{ns}}$ & $0,257^{\mathrm{ns}}$ & $0,146^{\mathrm{ns}}$ & $0,797^{\mathrm{ns}}$ \\
\hline II $\mathrm{x}$ III & $0,432^{\text {ns }}$ & $0,383^{\mathrm{ns}}$ & $0,243^{\mathrm{ns}}$ & $10,162 *$ & $0,180^{\mathrm{ns}}$ & $0,802^{\mathrm{ns}}$ \\
\hline
\end{tabular}

ns = não-significativo a $95 \%$ de probabilidade $\mathrm{e}^{*}=$ significativo a $95 \%$ de probabilidade.

\section{CONCLUSÕES}

- A estrutura da floresta caracteriza-se por alta diversidade florística, poucas espécies dominantes e muitas espécies raras.

- As espécies de maior valor de importância relativa sem valor comercial ocuparam as primeiras posições na estrutura da floresta.

- As espécies Lecythis idatimon, Rinorea guianensis e Pouteria guianensis são importantes na estrutura da floresta, pois ocorreram em todos os níveis de inclusão e classes de estoque.

- A análise da estrutura em classes de estoque permitiu um melhor conhecimento da composição de espécies e da estrutura fitossociológica e das distribuições diamétrica e espacial das espécies, sendo útil em estudos fitossociológicos e na elaboração e execução de planos de manejo florestal sustentável para essa floresta.

- O conhecimento da estrutura diamétrica auxilia a condução da floresta, com uma estrutura balanceada, na determinação da intensidade de corte e na manutenção da capacidade de sustentação da produção, bem como no estabelecimento do ciclo de corte e na colheita de madeira.

- Com base no estoque comercial remanescente, a área em estudo pode ser sustentável no curto, médio e longo prazos, desde que as prescrições de manejo não sejam violadas.

- As unidades de amostras inventariadas resultaram em um limite de erro inferior ao erro máximo estabelecido para inventariar florestas nativas.

\section{REFERÊNCIAS BIBLIOGRÁFICAS}

AMARAL, P. et al. Floresta para sempre: um manual para a produção de madeira na Amazônia. Belém: 1998. 155 p.

ARAUjO, M. M. et al. Padrões de distribuição espacial de espécies florestais em fragmento de floresta estacional decidual, Santa Maria - RS, Brasil. In: SIMPÓSIO LATINO-AMERICANO SOBRE MANEJO FLORESTAL, 2000, Santa Maria. Anais... Santa Maria: 2000. p.345-360.

BARROS, A. V.; BARROS, P. L. C.; SILVA, L. C. B. Análise fitossociológica de uma floresta situada em Curuá-Una - Pará. Revista Ciências Agrárias, n. 34, p. 9-36, 2000.

BARROS, P. L. C. Estudo das distribuições diamétricas de florestas do Planalto Tapajós-PA. 1980. 123 f. Dissertação (Mestrado em Engenharia Florestal) - Universidade Federal do Paraná, Curitiba, 1980.

BOURGERON, P. S. Spatial aspects of vegetation structure. In: GOLLEY, F. B. (Ed.). Tropical rain forest ecosystems. Amsterdam: Elsevier Scientific, 1983. p.29-47.

BROWER, J. E.; ZAR, J. H. Field and laboratory methods for general ecology. Dubuque: Wm. C. Brown Publishers, 1984. 226 p.

CARVALHO, J. O. P.; LOPES, J. C. A.; SILVA, J. N. M. Dinâmica da diversidade de espécies em uma floresta de terra firme na Amazônia brasileira relacionada à intensidade de exploração. In: SIMPÓSIO SILVICULTURAL NAAMAZÔNIA ORIENTAL, 1999, Belém. Resumos expandidos... Belém: EMBRAPA-CPATU/DFID, 1999. p. 167-173. (EMBRAPA-CPATU. Documentos, 123).

R. Árvore, Viçosa-MG, v.30, n.1, p.75-87, 2006 
CHICHORRO, J. F. Análise estrutural e econômica de multiprodutos de madeira em florestas naturais. 2000. 241

f. Tese (Doutorado em Ciência Florestal) Universidade Federal de Viçosa, Viçosa, 2000.

IBAMA. Portaria no 06-N. Diário Oficial da União, Brasília, de 03 de abril de 1992. 4p.

IBAMA. Instrução normativa no 6. Diário Oficial da União, Brasília, de 28 de dezembro de 1998. 6p.

FINOL, U. V. H. Nuevos parametros a considerarse en el analises estructural de las Selvas Virgines tropicais. Revista Florestal Venezolana, v. 14, n. 21, p. 29-42, 1971.

GOMES, A. P. C. Critérios e indicadores de sustentabilidade para o manejo de florestas tropicais. 2000. 109 f. Dissertação (Mestrado em Ciência Florestal) - Universidade Federal de Viçosa, Viçosa, 2000.

GRAYBILL, F. A. Theory and application of the linear model. Massachusetts: Ouxburg Press, 1976. 704 p.

KELMAN, M. C. Plant geography. London: 1975. 135 p.

LAMPRECHT, H. Silvicultura nos Trópicos: ecossistemas florestais e respectivas espécies arbóreas possibilidades e métodos de aproveitamento sustentado. Eschborn: Deustsche Gessells chaft für technisch Zusammenarbeti (GTZ) GmbH, 1990. 343 p.

LIMA FILHO, D. A. et al. Inventário florístico de floresta ombrófila densa de terra firme, na região do rio Urucu-Amazonas, Brasil. Acta

Amazônica, v. 31, n. 4, p. 565-579, 2001.

LONGHI, S. J. A estrutura de uma floresta natural de Araucaria angustifolia (Ber.) O.Ktze, no sul do Brasil. 1980. $198 \mathrm{f}$. Dissertação (Mestrado em Ciência Florestal) Universidade Federal do Paraná, Curitiba, 1980.
MACIEL, M. N. M.; QUEIROZ, W. T.; OLIVEIRA, F. A. Parâmetros fitossociológicos de uma floresta tropical de terra firme na Floresta Nacional de Caxiuanã-PA. Revista Ciências Agrárias, n. 34, p. 85-106, 2000.

MARTins PInTO, A. C. Análise de danos de colheita de madeira em floresta tropical úmida sob regime de manejo florestal na Amazônia Ocidental. 2000. 165 f. Dissertação (Mestrado em Ciência Florestal) - Universidade Federal de Viçosa, Viçosa, 2000.

MEYER, H. A. Structure, growth, and drain in balanced uneven-aged forests. Journal of Forestry, v.50, p.85-92, 1952.

MUELLER-DOMBOIS, D.; ELLEMBERG, H. Aims and methods of vegetation ecology. New York: Jonh Wiley Sons, 1974. 547 p.

QUEIROZ, W. T. Efeitos da variação estrutural em unidades amostrais na aplicação do processo de amostragem por conglomerados nas florestas do Planalto do Tapajós. 1997. 109 f.

Dissertação (Mestrado em Engenharia Florestal) Universidade Federal do Paraná, Curitiba, 1997.

RIBEIRO, R. J. et al. Estudo fitossociológico nas regiões de Carajás e Marabá - PA, Brasil. Acta Amazônica, v.29, n.2, p.207-222, 1999.

SILVA, J. N. M.; LOPES, J. C. A. Inventário florestal contínuo em florestas tropicais: a metodologia utilizada pela EMBRAPA-CPATU na Amazônia brasileira. Belém: EMBRAPA-CPATU, 1984. 39 p.

SOUZA, A. L. Estrutura, dinâmica e manejo de florestas tropicais. Viçosa: Universidade Federal de Viçosa, 2003. 122 p. (Notas de aula).

SOUZA, D. R. Sustentabilidade ambiental e econômica do manejo em floresta ombrófila densa de terra firme, Amazônia Oriental. 2003. 123 f. Tese (Doutorado em Ciência Florestal) - Universidade Federal de Viçosa, Viçosa, 2003.

SOUZA, A. L.; JESUS, R. M. Distribuição diamétrica de espécies arbóres da Floresta Atlântica: análise de agrupamento. Viçosa, SIF, 1994. 30 p. 
YARED, J. A. G.; COUTO, L.; LEITE, H. G.

Composição florística de florestas secundária e primária, sob efeito de diferentes sistemas silviculturais, na Amazônia Oriental. Revista Árvore, v. 22, n. 4, p. 463-474, 1998.
YARED, J. A. G.; COUTO, L.; LEITE, H. G.

Diversidade de espécies em florestas secundária e primária, sob efeito de diferentes sistemas silviculturais, na Amazônia Oriental. Revista Árvore, v. 24, n. 1, p. 83-90, 2000. 